The Version of Record of this manuscript has been published and is available in

Journal of Mathematics and Music, May 2019

https://doi.org/10.1080/17459737.2019.1608600

\title{
Harmonic distance in intervals and chords
}

\author{
Rafael Cubarsi \\ Departament de Matemàtiques, Universitat Politècnica de Catalunya, Barcelona, Spain
}

\begin{abstract}
The harmonic distance between two pure tones, in the sense used by Tenney, is generalised to chords whose pitches are harmonic fractions. In the tonal graph generated by the harmonics involved in a chord, which for n-TET systems has its equivalent in the Tonnetz, the melodic distance between the lowest common ancestor and the lowest common harmonic of the pitches composing the chord is a measure of the relative dissonance. This notion, rooted in just intonation, is extended to Pythagorean tuning and is used as an approximation for equal temperament scales. Harmonic distance and sensory dissonance are compared and discussed for chords in different tuning systems. It is borne out that proximity of chords in the Tonnetz is not exactly related to the harmonic distance.
\end{abstract}

Keywords: melodic distance; harmonic distance; chords; consonance; dissonance; Tonnetz; tuning systems

2010 Mathematics Subject Classification: 05C12; 11A05; 54E35

\section{Introduction}

Helmholtz (1863) used a device called resonator to hear out the overtones of a complex tone and quantify the dissonance in regard to the beatings. He showed that there is a critical bandwidth in the frequencies of two interfering waves that corresponds to a minimum degree of consonance. This value is not always proportional to the average of the interfering frequencies, but for low frequencies it remains almost constant. For a constant difference between the frequencies of the interfering waves, the dissonance is more evident as we move towards higher tones. For nearby frequencies, he estimated in approximately $30-40 \mathrm{~Hz}$ the critical bandwidth in which the sensation is more uncomfortable. Below $30 \mathrm{~Hz}$, tones tend to merge and, therefore, to be consonants. Above $40 \mathrm{~Hz}$, they return slowly to consonance.

By varying continuously the frequency of two interfering waves, Helmholtz noticed that frequencies maintaining a rational proportion are perceived by the ear as an increase of consonance. For the $p$-th and $q$-th harmonics of a fundamental tone $\nu_{0}$, with their ratio satisfying $1 \leq p / q<2$, the degree of consonance can be quantified from approximately the same ratio $p / q$, so that the smaller the ratio the smaller the consonance (by excluding the value 1). In Table 1, Helmholtz's $(1863,187)$ table with the ratios of the main harmonics and the intensity of influence $\left(C_{H}\right.$, in $\%)$ is reproduced. This parameter, used to sort the table, is the relative strength of the beats resulting from the mistuning of the corresponding interval, obtained by modelling the strength of the sympathetic vibration produced in the Corti's organ (ibid. Appendix 15, 415). It results in an amount

$$
C_{H}(p, q)=\frac{100}{p q} .
$$

Email: rafael.cubarsi@upc.edu 


\begin{tabular}{c|c|c} 
ratio & $C_{H}$ & $\Delta_{H}$ \\
\hline \hline $1 / 1=1.000$ & 100 & 0.0 \\
$2 / 1=2.000$ & 50 & 1.0 \\
$3 / 2=1.500$ & 16.7 & 2.6 \\
$4 / 3=1.333$ & 8.3 & 3.6 \\
$5 / 3=1.667$ & 6.7 & 3.9 \\
$5 / 4=1.250$ & 5 & 4.3 \\
$7 / 4=1.750$ & 3.6 & 4.8 \\
$6 / 5=1.200$ & 3.3 & 4.9 \\
$7 / 5=1.400$ & 2.8 & 5.2 \\
$8 / 5=1.600$ & 2.5 & 5.3 \\
$7 / 6=1.167$ & 2.4 & 5.4 \\
$9 / 5=1.800$ & 2.2 & 5.5 \\
$8 / 7=1.143$ & 1.8 & 5.8 \\
$9 / 7=1.286$ & 1.6 & 6.0 \\
$9 / 8=1.125$ & 1.4 & 6.2
\end{tabular}

Table 1. Helmholtz's table for the main harmonic ratios within an octave and the relative strength of the beats resulting from the mistuning of the corresponding interval $\left(C_{H}\right.$, in \%). The column on the right for $\Delta_{H}$ estimates the reciprocal of $C_{H}$ in a logarithmic scale (dimensionless). In gray, ratios involving harmonics of order greater than or equal to 7.

Helmholtz argued that the seventh harmonic sounds very low in most instruments and that higher harmonics generally have a negligible presence. Then, without taking into account ratios of harmonics involving the seventh and higher (marked in gray in Table 1), with exception of the ratio $5 / 3$, it holds the approximate rule ${ }^{1}$ that the quotient maintains a direct relation to $C_{H}$. The table is extended with a column showing an inverse relation to $C_{H}$ in logarithmic scale (therefore, a dimensionless quantity),

$$
\Delta_{H}(p, q)=\log _{2} \frac{100}{C_{H}(p, q)}=\log _{2}(p q) \in[0, \infty)
$$

that matches Tenney's harmonic distance for pure tones Tenney (2015, 240-279). According to Tenney $(2015,280-304)$, tones represented by proximate points in the harmonic space tend to be heard as being in a consonant relation to each other, while tones represented by more widely separated points are heard as mutually dissonant, even beyond the degree of beatings. Thus, the harmonic distance indicates an important correlation between consonance and dissonance. In Tenney's approach, harmonic distance is measured for pairs of tones. Here, it will be generalised to an arbitrary number of tones and will be called harmonic dissonance.

The theory of roughness ${ }^{2}$ has its grounds in Helmholtz's work. Plomp and Levelt (1965) anal-

\footnotetext{
${ }^{1}$ In the history of the problem of consonance the coincidence theory was the first one providing a precise quantitative link between musical and acoustical phenomena (Cohen 1985). The theory was established by Isaac Beeckman (in 1614-1615) and was further elaborated by Descartes, Mersenne, and Galileo, by relating the degree of consonance with the product $p q$.

${ }^{2}$ Roughness theory only provides a partial explanation for the sensations of consonance and dissonance, since they may also be sensed in intervals formed by tones with only few or even no interfering overtones, i.e., roughness is a matter of loudness, consonance is a matter of pitch; but loudness and pitch are independent perceptional moments. Modern psychoacoustics shows that the relation between beatings and roughness is complex and not a linear one (Fastl and Zwicker 2007, 257-264). Alternatively, Stumpf (1890) explained psychoacoustic consonance and dissonance in terms of perceptual fusion, i.e., the tendency for some sound combinations to cohere into a single sound image. He suggested that fused sounds are generally consonant, while segregated sounds are dissonant. Tonal fusion has a neurophysiological basis (Langner 2015) and is probably the decisive basis of the concept of consonance (Ebeling 2008). Stolzenburg (2015) shows how recent results from psychophysics and neuroacoustics provide a consistent computational theory of consonance/dissonance perception. In addition to the historical perspective of Tenney (1988), the reader is also referred to Mazzola, Göller, and Müller (2012, Appendix B), who offers a discussion on symbolic, physiological and psychological aspects of consonance and dissonance. They conclude: "it is hardly astonishing that psychological and physiological layers are not congruent: what the ear (in Helmholtz' model) does not 'like' can very well be 'agreeable' for the limbic system or the auditory cortex".
} 
ysed several explanations of consonance: frequency ratio, relationship of harmonics, beats between harmonics, difference tones, and fusion. According to these authors, the tonal consonance is mainly determined by the roughness of rapid beats, although dependence of the roughness on the critical band requires a modification of Helmholtz's criterion for maximal roughness, because the critical bandwidth is not equally wide at all frequencies. From a psychophysiological point of view, roughness is a sensation related to the inability to discriminate different pitches whose frequency difference is smaller than the critical bandwidth. The bandwidth of the zone of minimum consonance increases progressively from $1000 \mathrm{~Hz}$ to higher frequencies, although the critical bandwidth of the minimum occupies the same relative position.

In this context, it is possible to get a model for the sensory dissonance of a set of tones, not necessarily harmonics or subharmonics of a fundamental tone. The most extended model (Sethares 1998) approximates the function of dissonance, obtained by Plomp and Levelt experimentally, to measure the average dissonance of two tones. This model is used for a sound consisting of an arbitrary set of non-two equal tones $F=\left\{\nu_{1}, \ldots, \nu_{n}\right\}$, which is called spectrum, as for chords or several instruments playing simultaneously. The sensory dissonance $\Delta_{S}(F)$ produced by the spectrum interfering with itself is approached additively from the weighted sum of their functions of dissonance for couples of tones with regard to their amplitudes $A=\left\{a_{1}, \ldots, a_{n}\right\}$. The resulting curves of sensory dissonance (such as those of Figure 7) show relative minima in the ratios that provide a greater sensation of consonance.

In the present work, the harmonic distance will be generalised to chords as an estimation of the degree of beatings produced by a set of tones maintaining rational proportions. In addition, it will be obtained according to a geometrical interpretation, by placing the tones on a tonal graph and measuring the melodic distance between their lowest common ancestor (LCA), that assumes the role of missing fundamental, and their lowest common harmonic (LCH), the lowest frequency of which they are subharmonics.

\section{Melodic distance}

Hearing, like the other senses, perceive the relative changes of stimulus. In particular, it detects relative variations of a frequency $\nu \in \Omega \equiv(0, \infty)$. In a differential approach, in order to measure variations of frequency we can use a variable $I$ defined from the following proportionality,

$$
\mathrm{dI}=\mathrm{a} \frac{\mathrm{d} \nu}{\nu} ; \mathrm{a} \in \mathbb{R} \Rightarrow \mathrm{I}\left(\nu, \nu_{0}\right)=\mathrm{a} \ln \frac{\nu}{\nu_{0}} .
$$

Then, $I\left(\nu, \nu_{0}\right)$ measures the interval of frequency between $\nu$ and an arbitrary initial value $\nu_{0}$ in a logarithmic scale. By imposing that the unit interval corresponds to one octave, i.e., $I\left(2 \nu_{0}, \nu_{0}\right)=$ 1 , we get $a=\frac{1}{\ln 2}$. Hence, the interval between two pure tones is obtained as follows

$$
I\left(\nu, \nu_{0}\right)=\log _{2} \frac{\nu}{\nu_{0}}
$$

satisfying $I\left(\nu, \nu_{0}\right)=-I\left(\nu_{0}, \nu\right)$. Therefore, the interval function has a sign and a direction.

On the other hand, its absolute value defines a melodic distance in $\Omega$ as

$$
d\left(\nu, \nu_{0}\right)=\left|I\left(\nu, \nu_{0}\right)\right|
$$

For a fixed $\nu_{0}$, the interval function and the melodic distance are defined in the domain $(0, \infty)$ of frequencies (frequency ratios, in general) and take values in the space of their logarithms, i.e., in $(-\infty, \infty)$ the former, for measurements of intervals between pitches, and in $(0, \infty)$ the latter.

An equivalence relation can be established if the frequencies $\nu$ and $2 \nu$ are identified as the same 
note, although the latter is an octave higher than the former. Thus, for a given value $\nu$, all the frequencies $2^{k} \nu, k \in \mathbb{Z}$, define one equivalence class. We assume the frequencies $\nu \in[1,2)$ as the representative of their frequency classes (FCs). Therefore, if we use the appropriate unit system so that $\nu_{0}=1$ is the fundamental frequency ratio, the set of all the octaves of the fundamental is a cyclic subgroup of $\Omega$ for multiplication, denoted as $\Omega^{2}=\left\{2^{k}, k \in \mathbb{Z}\right\}$, and the FCs are the elements of the quotient group $\Omega_{0}=\Omega / \Omega^{2}$. Then, the notes or pitch classes, i.e. the FCs in a logarithmic scale, are obtained as the fractional part $\left\{\log _{2} \frac{\nu}{\nu_{0}}\right\}$ and are generated by the equivalence relation $S_{0}=\mathbb{R} / \mathbb{Z}$.

We write the interval from the fundamental to a frequency $\nu$ as

$$
I(\nu) \equiv I(\nu, 1)
$$

Similarly, $|I(\nu)|$ is the distance between $\nu$ and the fundamental. As intervals have the octave as unit length, the integer part (or floor function)

$$
\lfloor I(\nu)\rfloor=\left\lfloor\log _{2} \nu\right\rfloor
$$

indicates the octave where the frequency $\nu$ is placed, with origin in the fundamental. For instance, given a value $h>0$, the frequency $\nu=h^{p}$ is placed at an interval $I(\nu)=p \log _{2} h$ from the fundamental and $\left\lfloor p \log _{2} h\right\rfloor$ is the index of octave corresponding to $h^{p}$. On the other hand, the note associated with $\nu \in \Omega$ is the fractional part

$$
\{I(\nu)\}=\left\{\log _{2} \nu\right\} \in S_{0}
$$

If $\nu \in \Omega_{0}$, i.e., is a FC, it is not necessary to write the curly brackets.

According to the notation of equation (6) it is satisfied

$$
I\left(\nu_{1} \nu_{2}\right)=I\left(\nu_{1}\right)+I\left(\nu_{2}\right)
$$

and equation (4) can be expressed as

$$
I\left(\nu, \nu_{0}\right)=I(\nu)-I\left(\nu_{0}\right)
$$

For example, a sampling of the octave with frequencies according to the 12-tone equal temperament (TET) scale gives FCs $\alpha_{k}=2^{\frac{k}{12}} ; k=0, \ldots, 11$. They are in geometric progression in $\Omega_{0}$ and their notes $I\left(\alpha_{k}\right)=\frac{k}{12} \in S_{0}$ are in arithmetic progression. In a $n$-TET scale $E_{n}^{T}=\left\{2^{\frac{k}{n}}, k \in \mathbb{Z}_{n}\right\}$, with $n>1$, the notes of equation (8) have a symmetrical distribution in $S_{0}$.

If we would think of the octave $\Omega_{0}$ as the interval $[1,2) \in \mathbb{R}$, then the melodic interval between two FCs, namely $I\left(\nu, \nu^{\prime}\right)=I(\nu)-I\left(\nu^{\prime}\right)$, would have upper bound $I(2,1)=1$. Under this assumption, this maximum value can only be achieved for FCs tending to the fundamental by the respective extremes of $[1,2)$. But, actually in $\Omega_{0}$ these FCs are arbitrarily close to each other.

If we deal with FCs in $\Omega_{0}$, with both extremes of $[1,2)$ identified, the function $|I|$ does not meet the properties of a distance. For example, the absolute value of the interval ranging from $\frac{3}{2}$ to the fundamental 1 is $\log _{2} \frac{3}{2}$ or $\log _{2} \frac{4}{3}$, depending on whether we look to the left or the right. The distance should indicate the minimum interval, in absolute value, between two FCs. Therefore, in the octave $\Omega_{0}$, as for a circle, the distance between two FCs $\alpha, \beta$ must be defined as

$$
d_{0}(\alpha, \beta)=\min (|I(\alpha, \beta)|, 1-|I(\alpha, \beta)|) .
$$

In other words, we consider the quotient metric in $\mathbb{R} / \mathbb{Z}$. 


\section{Distance between coprime harmonics}

A geometrical representation on a tonal graph will be used to relate the dissonance between two harmonics of the same fundamental to a certain melodic distance.

Suppose we compare two harmonics of a certain fundamental tone $\nu_{0}$,

$$
\nu_{p}=p \nu_{0} ; \quad \nu_{q}=q \nu_{0}
$$

Firstly we shall assume that $p$ and $q$ are positive coprime integers. Then, the prime factorisations $p=p_{1} \cdots p_{m}$ and $q=q_{1} \cdots q_{n}$ (they are unique) do not have any factor in common. Their greatest common divisor is $\operatorname{gcd}(p, q)=1$, and their least common multiple is $\operatorname{lcm}(p, q)=p q$. In this situation, the smaller natural numbers $a, b$ for which a harmonic of $\nu_{p}$ matches one of $\nu_{q}$, i.e.,

$$
a p \nu_{0}=b q \nu_{0},
$$

obviously are $a=q$ and $b=p$. Therefore, the lowest common harmonic (LCH) of these two tones is

$$
\operatorname{lch}\left(\nu_{p}, \nu_{q}\right)=p q \nu_{0}=\operatorname{lcm}(p, q) \nu_{0} .
$$

In one dimension, according to equations (5) and (6), the melodic distance between $\nu_{0}$ and $\operatorname{lch}\left(\nu_{p}, \nu_{q}\right)$ is

$$
d\left(p q \nu_{0}, \nu_{0}\right)=I(p q)=\log _{2} p q .
$$

We now build the directed tonal graph of Figure 1, with frequencies in the nodes that are connected by their ratios in the edges, denoted as $p$ in one direction and $q$ in the other. We shall use this graph ${ }^{3}$ to measure distances between harmonics expressed as $p^{m} q^{n} \nu_{0} ; m, n \in \mathbb{N}$.

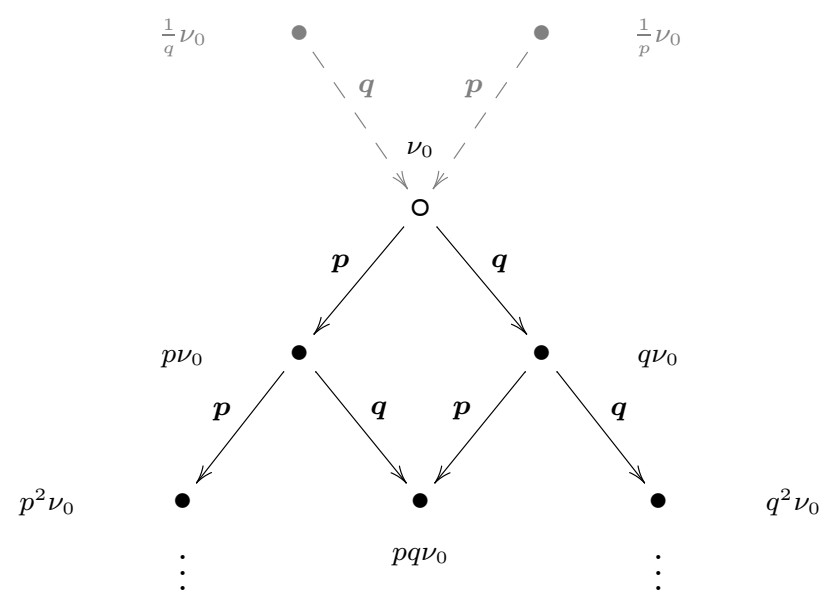

Figure 1. Tonal graph of coprime edges $p, q$ with lowest common ancestor $\nu_{0}$. The ancestors of $\nu_{0}$ are depicted in gray.

By starting in the node $\nu_{0}$, along the $p$ direction from top to bottom, we meet the subset of harmonics $p \nu_{0}, p^{2} \nu_{0}$, etc., and along the $q$ direction the subset $q \nu_{0}, q^{2} \nu_{0}$, etc. The melodic distance of two contiguous nodes is $\log _{2} p$ if they are connected by an edge $p$, and $\log _{2} q$ if they are connected by an edge $q$.

\footnotetext{
${ }^{3}$ It is similar to the tonal network or Euler's Tonnetz with edges that, in equal temperament, are intervals between pitches instead of ratios, but it has not the orbifold structure induced by the pitch classes.
} 
It is possible to go from nodes $\nu_{0}$ to $p^{m} q^{n} \nu_{0}$ by any path that contains $m p$-edges and $n q$-edges, regardless of the order. The melodic distance of this path is

$$
d\left(p^{m} q^{n} \nu_{0}, \nu_{0}\right)=d\left(p^{m} \nu_{0}, \nu_{0}\right)+d\left(q^{n} \nu_{0}, \nu_{0}\right)=I\left(p^{m}\right)+I\left(q^{n}\right)=m \log _{2} p+n \log _{2} q,
$$

which can be calculated as the sum of distances from the common origin $\nu_{0}$ to $\nu_{p}=p^{m} \nu_{0}$ and to $\nu_{q}=q^{n} \nu_{0}$, respectively. This common origin, in directed acyclic graphs is known as lowest common ancestor (LCA), which is the lowest node in the tonal graph of edges $p, q$ that has both $\nu_{p}$ and $\nu_{q}$ as descendants. Therefore,

$$
\nu_{0}=\operatorname{lca}\left(p^{m} \nu_{0}, q^{n} \nu_{0}\right) ; \quad m, n \in \mathbb{N} .
$$

Notice that, in all the nodes of Figure 1, the common factor is the LCA. Then, expressions referring to distance are equally valid if the LCA is taken as unit.

Thus, for any $p$ and $q$ coprime (it is also valid for the powers $p^{m}$ and $q^{n}$ ), the harmonic distance between the tones $\nu_{p}=p \nu_{0}$ and $\nu_{q}=q \nu_{0}$ is defined as the following melodic distance

$$
d_{H}\left(\nu_{p}, \nu_{q}\right) \equiv d\left(\operatorname{lca}\left(\nu_{p}, \nu_{q}\right), \operatorname{lch}\left(\nu_{p}, \nu_{q}\right)\right) \text {, }
$$

which is equivalent to the sum of the melodic distances from lca $\left(\nu_{p}, \nu_{q}\right)$ to $\nu_{p}$ and to $\nu_{q}$,

$$
d_{H}\left(\nu_{p}, \nu_{q}\right)=d\left(p q \nu_{0}, \nu_{0}\right)
$$

For all $\nu_{0} \in \mathbb{R}$, the following relationship is satisfied

$$
d_{H}\left(p \nu_{0}, q \nu_{0}\right)=d_{H}(p, q)
$$

This value is the distance ${ }^{4}$ on the tonal graph from the common fundamental of both frequencies up to the closest frequency of which they are subharmonics.

The fact that the harmonics $p \nu_{0}, q \nu_{0}$ and $p q \nu_{0}$ have common periods with the wave of frequency $\nu_{0}$ can be interpreted according to the forced harmonic oscillator model (e.g., Mickens 1981), where the mutual dependency of co-existing sounds can be traced. A perturbing vibration of frequency $\nu_{0}$ elicits periodic solutions with free oscillations of frequencies $p \nu_{0}, q \nu_{0}$ and $p q \nu_{0}$, which are its superharmonics. Similarly, the harmonic $p q \nu_{0}$ has a period that coincides with periods of the subharmonics $p \nu_{0}$ and $q \nu_{0}$. For instance, a superposition of waves of frequencies $1,2,3,6$ is a periodic solution of the forced harmonic oscillator of period $2 \pi$, being the frequency 1 the highest common subharmonic and the frequency 6 is lowest common superhamonic. Thus, for many of the harmonics of a fundamental frequency it is not possible to identify to which single wave they belong. Therefore, the LCH can be associated with a fused sound image where its subhamonics become immersed. The LCA is the common fundamental.

The value $d_{H}(p, q)$ of equation (15) matches equation (2) used to calculate the values on the right-hand side column ${ }^{5}$ of Table 1 . Therefore, quoting Tenney, the harmonic distance $d_{H}(p, q)$ might be considered as a possible correlate of the relative dissonance of the combined spectra of the harmonic series of these sounds.

The structure of directed graph of Figure 1 may also be read from bottom to top, with ratios $\frac{1}{p}$ and $\frac{1}{q}$ between consecutive nodes. It can be easily generalised for more than two coprime harmonics, by adding a new dimension for each new harmonic.

\footnotetext{
${ }^{4}$ Of course, the properties of a distance are fulfilled, i.e., $d_{H}(p, q) \geq 0 ; \quad d_{H}(p, q)=0 \Longleftrightarrow p=q ; d_{H}(p, q)=d_{H}(q, p)$; $d_{H}(p, q)+d_{H}(q, r) \geq d_{H}(p, r)$. In the last inequality, only when $q=1$ it is hold that $d_{H}(p, 1)+d_{H}(1, r)=d_{H}(p, r)$.

${ }^{5}$ Helmholtz's values for $C_{H}$, which are given with one decimal accuracy (Table 1), match those given by this formula, except the ratio $5 / 7$, which should be 2.9 instead of 2.8 , as published in his book, resulting from rounding 2.86 .
} 


\section{Distance between arbitrary harmonics}

We now compare two non-coprime harmonics of a fundamental tone $\nu_{0}$,

$$
\nu_{P}=P \nu_{0} ; \quad \nu_{Q}=Q \nu_{0}
$$

with $P, Q \in \mathbb{N}$, such that

$$
D \equiv \operatorname{gcd}(P, Q)>1
$$

We write $\nu_{P}=p D \nu_{0}$ and $\nu_{Q}=q D \nu_{0}$, where $p$ and $q$ are coprime, as in the previous section. Having in mind that

$$
\operatorname{gcd}(P, Q) \operatorname{lcm}(P, Q)=P Q
$$

we write

$$
M \equiv \operatorname{lcm}(P, Q)=p q D .
$$

Then, in a similar way as in equation (12), we get the lowest common harmonic as

$$
\operatorname{lch}\left(\nu_{P}, \nu_{Q}\right)=p q D \nu_{0}=\operatorname{lcm}(P, Q) \nu_{0},
$$

and the lowest common ancestor as

$$
\operatorname{lca}\left(\nu_{P}, \nu_{Q}\right)=\operatorname{gcd}(P, Q) \nu_{0} .
$$

Therefore, we get the same graph structure of Figure 1, but now with the common origin $D \nu_{0}$. It is now straightforward to derive the expression for the harmonic distance. As in equation (15), the harmonic distance is calculated as the melodic distance from lca $\left(\nu_{P}, \nu_{Q}\right)$ to $\operatorname{lch}\left(\nu_{P}, \nu_{Q}\right)$, i.e.,

$$
d_{H}\left(\nu_{P}, \nu_{Q}\right)=d\left(M \nu_{0}, D \nu_{0}\right)=d\left(p q D \nu_{0}, D \nu_{0}\right)=I(p q)
$$

so that we get the following equivalences

$$
d_{H}\left(\nu_{P}, \nu_{Q}\right)=d_{H}(P, Q)=d_{H}(p, q)=\log _{2} p q .
$$

Therefore, the harmonic distance is in this case defined as

$$
d_{H}\left(\nu_{P}, \nu_{Q}\right)=\log _{2} \frac{\operatorname{lch}\left(\nu_{P}, \nu_{Q}\right)}{\operatorname{lca}\left(\nu_{P}, \nu_{Q}\right)}=\log _{2} \frac{\operatorname{lcm}(P, Q)}{\operatorname{gcd}(P, Q)},
$$

which is satisfied for any $\nu_{0} \in \mathbb{R}$.

The last expression agrees with the harmonic distance for pure tones used by Tenney (2015, 240-279) in his 1979 The Structure of Harmonic Series Aggregates. In the following sections we shall generalise it for tones obtained as fractions of harmonics and for sets of more than two tones.

Note that, according to equation (19), for the two arguments on the left-hand side member of equation (20) we have lca $\left(p D \nu_{0}, q D \nu_{0}\right)=D \nu_{0}$; for the middle ones, $\operatorname{lca}(P, Q)=D$; and for the right-hand side member, lca $(p, q)=1$.

We shall say that the respective graphs are equivalent, since they only differ in their fundamental tone of reference, namely $D \nu_{0}, D$, and 1, respectively. 
Therefore, the harmonic distance does not change if both arguments are multiplied by a same non-null factor $x \in \mathbb{R}$,

$$
\operatorname{gcd}(p, q)=1 \Longrightarrow d_{H}(p x, q x)=d_{H}(p, q) ; \quad \operatorname{lca}(p x, q x)=x
$$

and, in general,

$$
\operatorname{lca}\left(\nu_{P} x, \nu_{Q} x\right)=\operatorname{lca}\left(\nu_{P}, \nu_{Q}\right) x=\operatorname{gcd}(P, Q) \nu_{0} x .
$$

The property of equation (22) allows us to calculate the harmonic distance between the two tones $\nu_{P}$ and $\nu_{Q}$ as the distance between their ratio and the unity,

$$
d_{H}\left(\nu_{P}, \nu_{Q}\right)=d_{H}\left(\frac{\nu_{P}}{\nu_{Q}}, 1\right)=d_{H}(p, q)=d_{H}\left(\frac{p}{q}, 1\right) .
$$

\section{Distance between harmonic fractions}

The property equation (22) also allows to calculate the harmonic distance between two tones expressed as irreducible fractions of harmonics, namely $p / q$ and $r / s$. Then, owing to equations (21) and (22), we have

$$
d_{H}\left(\frac{p}{q}, \frac{r}{s}\right)=d_{H}\left(\frac{p s}{q r}, 1\right)=d_{H}(p s, q r)=\log _{2} \frac{\operatorname{lcm}(p s, q r)}{\operatorname{gcd}(p s, q r)}
$$

and, by equation (23), we obtain the respective lowest common harmonics

$$
\operatorname{lca}\left(\frac{p}{q}, \frac{r}{s}\right)=\frac{1}{q s} \operatorname{gcd}(p s, q r) ; \quad \operatorname{lca}\left(\frac{p s}{q r}, 1\right)=\frac{1}{q r} \operatorname{gcd}(p s, q r) ; \quad \operatorname{lca}(p s, q r)=\operatorname{gcd}(p s, q r) .
$$

These calculations can be simplified, and afterwards generalised to more than two tones, by taking advantage of the extended notion of least common multiple and greatest common divisor for rational numbers, provided they are irreducible fractions. Thus, we write

$$
\operatorname{lcm}\left(\frac{p}{q}, \frac{r}{s}\right)=\frac{\operatorname{lcm}(p, r)}{\operatorname{gcd}(q, s)} ; \quad \operatorname{gcd}\left(\frac{p}{q}, \frac{r}{s}\right)=\frac{\operatorname{gcd}(p, r)}{\operatorname{lcm}(q, s)}
$$

Then, equation (25) becomes a particular case of equation (21),

$$
d_{H}\left(\frac{p}{q}, \frac{r}{s}\right)=\log _{2} \frac{\operatorname{lcm}\left(\frac{p}{q}, \frac{r}{s}\right)}{\operatorname{gcd}\left(\frac{p}{q}, \frac{r}{s}\right)}
$$

and, by taking into account equation (27),

$$
d_{H}\left(\frac{p}{q}, \frac{r}{s}\right)=\log _{2}\left(\frac{\operatorname{lcm}(p, r)}{\operatorname{gcd}(p, r)} \frac{\operatorname{lcm}(q, s)}{\operatorname{gcd}(q, s)}\right) .
$$

Hence, we get

$$
d_{H}\left(\frac{p}{q}, \frac{r}{s}\right)=d_{H}(p, r)+d_{H}(q, s)
$$

As a corollary of the previous result, we will draw some consequences: 
(1) The calculation of the lowest common ancestor and the lowest common harmonic becomes a particular case of equations (18) and (19),

$$
\begin{aligned}
& \operatorname{lca}\left(\frac{p}{q}, \frac{r}{s}\right)=\operatorname{gcd}\left(\frac{p}{q}, \frac{r}{s}\right)=\frac{\operatorname{gcd}(p, r)}{\operatorname{lcm}(q, s)} \\
& \operatorname{lch}\left(\frac{p}{q}, \frac{r}{s}\right)=\operatorname{lcm}\left(\frac{p}{q}, \frac{r}{s}\right)=\frac{\operatorname{lcm}(p, r)}{\operatorname{gcd}(q, s)} .
\end{aligned}
$$

(2) The edges of the graph on the left-hand side of Figure 2 are coprime natural numbers, since they can be expressed as the ratios

$$
\begin{aligned}
& \alpha=\frac{p}{q} \frac{1}{\operatorname{lca}\left(\frac{p}{q}, \frac{r}{s}\right)}=\frac{p}{\operatorname{gcd}(p, r)} \frac{\operatorname{lcm}(q, s)}{q} ; \quad \beta=\frac{r}{s} \frac{1}{\operatorname{lca}\left(\frac{p}{q}, \frac{r}{s}\right)}=\frac{r}{\operatorname{gcd}(p, r)} \frac{\operatorname{lcm}(q, s)}{s} ; \\
& \beta^{\prime}=\frac{\operatorname{lch}\left(\frac{p}{q}, \frac{r}{s}\right)}{\frac{p}{q}}=\frac{\operatorname{lcm}(p, r)}{p} \frac{q}{\operatorname{gcd}(q, s)} ; \quad \alpha^{\prime}=\frac{\operatorname{lch}\left(\frac{p}{q}, \frac{r}{s}\right)}{\frac{r}{s}}=\frac{\operatorname{lcm}(p, r)}{r} \frac{s}{\operatorname{gcd}(q, s)} .
\end{aligned}
$$

(3) They satisfy $\alpha=\alpha^{\prime}$ and $\beta=\beta^{\prime}$, owing to the identities

$$
\frac{\operatorname{lcm}(q, s) \operatorname{gcd}(q, s)}{q s}=\frac{\operatorname{lcm}(p, r) \operatorname{gcd}(p, r)}{p r}=1 .
$$

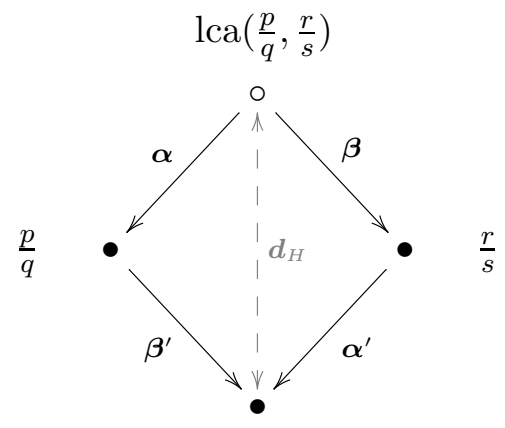

$\operatorname{lch}\left(\frac{p}{q}, \frac{r}{s}\right)$

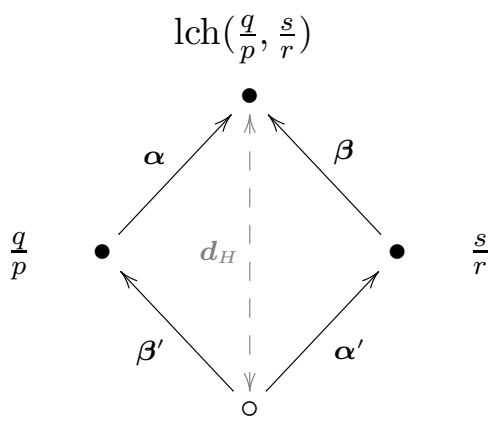

$\operatorname{lca}\left(\frac{q}{p}, \frac{s}{r}\right)$

Figure 2. (Left) Tonal graph of coprime edges $\alpha=\alpha^{\prime}$ and $\beta=\beta^{\prime}$ with lowest common ancestor lca $\left(\frac{p}{q}, \frac{r}{s}\right)$. (Right) Tonal graph for the inverse tones of the graph on the left.

To invert the ratios is equivalent to inverting the directions of the arrows, as shown on the right-hand side of Figure 2. In this case, according to equations (31) and (32) we get

$$
\operatorname{lca}\left(\frac{q}{p}, \frac{s}{r}\right)=\frac{1}{\operatorname{lch}\left(\frac{p}{q}, \frac{r}{s}\right)} ; \quad \operatorname{lch}\left(\frac{q}{p}, \frac{s}{r}\right)=\frac{1}{\operatorname{lca}\left(\frac{p}{q}, \frac{r}{s}\right)} .
$$

These expressions are also valid for a finite set of irreducible fractions. 


\section{Harmonic dissonance}

The use of equation (27) allows us to generalise the harmonic distance of equation (29) to a finite set of tones expressed as irreducible fractions, such as

$$
\Lambda=\left\{\lambda_{i}=\frac{p_{i}}{q_{i}} ; i \in I\right\}
$$

with $p_{i}, q_{i} \in \mathbb{N} \backslash\{0\}$ and $\operatorname{gcd}\left(p_{i}, q_{i}\right)=1$, as the melodic distance between their LCA and LCH. By considering separately the numerators and denominators of these fractions we define the sets

$$
\Pi=\left\{p_{i} ; i \in I\right\} ; \quad \Theta=\left\{q_{i} ; i \in I\right\} .
$$

Then, according to the definitions of equations (31) and (32), we have

$$
\begin{aligned}
& \operatorname{lca}(\Lambda)=\operatorname{gcd}(\Lambda)=\frac{\operatorname{gcd}(\Pi)}{\operatorname{lcm}(\Theta)}, \\
& \operatorname{lch}(\Lambda)=\operatorname{lcm}(\Lambda)=\frac{\operatorname{lcm}(\Pi)}{\operatorname{gcd}(\Theta)},
\end{aligned}
$$

so that the melodic interval between the above tones is

$$
d(\operatorname{lca}(\Lambda), \operatorname{lch}(\Lambda))=\log _{2} \frac{\operatorname{lcm}(\Lambda)}{\operatorname{gcd}(\Lambda)}=\log _{2} \frac{\operatorname{lcm}(\Pi)}{\operatorname{gcd}(\Pi)}+\log _{2} \frac{\operatorname{lcm}(\Theta)}{\operatorname{gcd}(\Theta)} .
$$

Therefore, this interval can be calculated separately as the sum of the respective intervals of the numerator and denominator of the fractions in $\Lambda$ as

$$
d(\operatorname{lca}(\Lambda), \operatorname{lch}(\Lambda))=d(\operatorname{lca}(\Pi), \operatorname{lch}(\Pi))+d(\operatorname{lca}(\Theta), \operatorname{lch}(\Theta)) .
$$

For a finite set of tones, the quantity

$$
\Delta_{H}(\Lambda) \equiv d(\operatorname{lca}(\Lambda), \operatorname{lch}(\Lambda))=\log _{2} \frac{\operatorname{lcm}(\Lambda)}{\operatorname{gcd}(\Lambda)}
$$

will be referred to as harmonic dissonance of the tones $\Lambda$. Thus, the harmonic dissonance $\Delta_{H}$ generalises the harmonic distance $d_{H}$ to more than two tones. Similarly as in equation (22), it is satisfied $\Delta_{H}(\Lambda)=\Delta_{H}(x \Lambda), \forall x \in \mathbb{R} \backslash\{0\}$. form

By considering equation (40), we express the harmonic dissonance in the following additive

$$
\Delta_{H}(\Lambda)=\Delta_{H}(\Pi)+\Delta_{H}(\Theta),
$$

which allows us to generalise, according to equation (1), the consonance as used by Helmholtz. Thus, for the tones $\Lambda$, the harmonic consonance is defined as

$$
C_{H}(\Lambda)=100 \frac{\operatorname{gcd}(\Lambda)}{\operatorname{lcm}(\Lambda)}=100 \frac{\operatorname{gcd}(\Pi)}{\operatorname{lcm}(\Pi)} \frac{\operatorname{gcd}(\Theta)}{\operatorname{lcm}(\Theta)} .
$$

It is worth noticing that the harmonic dissonance is not additive, i.e., it is not the sum of the dissonances between couples of tones, or between each tone and the fundamental tone. Instead, it is additive with regard to the numerators and denominators of tonal fractions. 


\section{Pythagorean tuning}

We now calculate harmonic distances between FCs of a Pythagorean scale, i.e., generated by the harmonics 2 and 3, obtained from ratios corresponding to octaves and fifths referred to the fundamental tone taken as unit.

We refer therefore to FCs of the form

$$
\nu_{p}=\frac{3^{p}}{2^{q}} ; \quad p, q \in \mathbb{N}
$$

satisfying $1 \leq \nu_{p}<2$. According to equation (7), for each $p$ (index of fifth), the value $q$ (index of octave) is totally determined as the integer part

$$
q=\left\lfloor p \log _{2} 3\right\rfloor .
$$

When calculating the harmonic distance of two tones $\nu_{p}=\frac{3^{p}}{2^{q}}$ and $\nu_{p^{\prime}}=\frac{3^{p^{\prime}}}{2^{q^{\prime}}}$, such that $p>p^{\prime}$ (this does not constrain the relative pitch of $\nu_{p}, \nu_{p^{\prime}}$ ), we must consider the relationship

$$
p \log _{2} 3>p^{\prime} \log _{2} 3 \Longrightarrow\left\lfloor p \log _{2} 3\right\rfloor \geq\left\lfloor p^{\prime} \log _{2} 3\right\rfloor \text {. }
$$

Therefore, according to equation (44),

$$
p>p^{\prime} \Longrightarrow q \geq q^{\prime}
$$

We now calculate the two nodes of the tonal graph

$$
\begin{aligned}
& \operatorname{lca}\left(\nu_{p}, \nu_{p^{\prime}}\right)=\operatorname{gcd}\left(\frac{3^{p}}{2^{q}}, \frac{3^{p^{\prime}}}{2^{q^{\prime}}}\right)=\frac{\operatorname{gcd}\left(3^{p}, 3^{p^{\prime}}\right)}{\operatorname{lcm}\left(2^{q}, 2^{q^{\prime}}\right)}=\frac{3^{p^{\prime}}}{2^{q}} ; \\
& \operatorname{lch}\left(\nu_{p}, \nu_{p^{\prime}}\right)=\operatorname{lcm}\left(\frac{3^{p}}{2^{q}}, \frac{3^{p^{\prime}}}{2^{q^{\prime}}}\right)=\frac{\operatorname{lcm}\left(3^{p}, 3^{p^{\prime}}\right)}{\operatorname{gcd}\left(2^{q}, 2^{q^{\prime}}\right)}=\frac{3^{p}}{2^{q^{\prime}}} ;
\end{aligned}
$$

with edges given by the positive integer ratios

$$
\begin{gathered}
\alpha=\frac{\nu_{p}}{\operatorname{lca}\left(\nu_{p}, \nu_{p^{\prime}}\right)}=3^{p-p^{\prime}} \\
\beta=\frac{\nu_{p^{\prime}}}{\operatorname{lca}\left(\nu_{p}, \nu_{p^{\prime}}\right)}=2^{q-q^{\prime}} .
\end{gathered}
$$

Finally, the harmonic dissonance between both tones is given by

$$
\Delta_{H}\left(\nu_{p}, \nu_{p^{\prime}}\right)=\log _{2}\left(3^{p-p^{\prime}} 2^{q-q^{\prime}}\right)=\left(p-p^{\prime}\right) \log _{2} 3+\left(q-q^{\prime}\right) .
$$

Notice that, by equation (45), the greater the difference $p-p^{\prime}$ the greater the harmonic dissonance. In other words, for fixed $p^{\prime}$ and $\nu_{p^{\prime}}$, the dissonance is an increasing function of $p$.

On the other hand, the function

$$
H_{3}(p) \equiv p \log _{2} 3+\left\lfloor p \log _{2} 3\right\rfloor
$$

is related to equation (3) so that, bearing in mind equation (44), it verifies

$$
H_{3}(p)=\Delta_{H}\left(\nu_{p}, 1\right)
$$




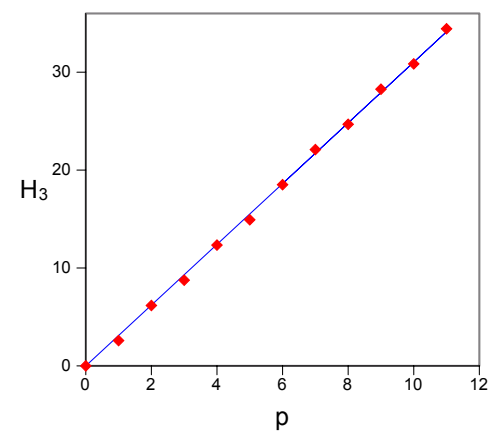

Figure 3. Approximation of the harmonic dissonance $\Delta_{H}\left(\nu_{p}, 1\right)$ (dots) between the tones of a Pythagorean scale and the fundamental tone by its regression line.

This function can be approximated by a regression line $y=k x$ around the points $x=0,1, \ldots, n-$ 1 corresponding to the $n$ notes of the scale considered. For the case $n=12$, the slope of the straight line is $k=3.10 \pm 0.02$, resulting in the adjustment of Figure 3. In a general case, we can write $H_{3}(p)=2 p \log _{2} 3-\varepsilon$, being $\varepsilon$ the fractional part $\left\{p \log _{2} 3\right\}$. We can assume that $\varepsilon$ is a random variable uniformly distributed in $[0,1)$ with expected value $\bar{\varepsilon}=\frac{1}{2}$. In such a case, the least squares approximation provides an estimate $6=2 \log _{2} 3-\frac{3}{2} \frac{1}{2 n-1}$, approaching $2 \log _{2} 3$ from below for large values of $n$. Therefore, the dissonance between two tones is approximately given by the difference

$$
\Delta_{H}\left(\nu_{p}, \nu_{p^{\prime}}\right)=H_{3}(p)-H_{3}\left(p^{\prime}\right) \approx k\left(p-p^{\prime}\right),
$$

from where we conclude that the difference of indices of fifth $p-p^{\prime}$ is a good way to measure the harmonic distance between pairs of tones of a Pythagorean scale.

It is interesting to investigate what happens if a new tone $\nu_{p^{\prime \prime}}=\frac{3^{p^{\prime \prime}}}{2 q^{\prime \prime}}$ satisfying $p>p^{\prime \prime}>p^{\prime}$ is added to the two previous tones. Notice that it does not mean that the new sound has an intermediate pitch. Then, the expressions of equation (46) do not change. Therefore, the harmonic dissonance of the set of three notes depends only on the extreme values of their indices $p$ of fifths,

$$
\Delta_{H}\left(\nu_{p}, \nu_{p^{\prime}}, \nu_{p^{\prime \prime}}\right)=\Delta_{H}\left(\nu_{p}, \nu_{p^{\prime}}\right) \Longleftrightarrow p>p^{\prime \prime}>p^{\prime}
$$

In this case, the tonal graph has a new edge, in a third dimension, with ratio

$$
\gamma=\frac{\nu_{p^{\prime \prime}}}{\operatorname{lca}\left(\nu_{p}, \nu_{p^{\prime}}\right)}=3^{p^{\prime \prime}-p^{\prime}} 2^{q-q^{\prime \prime}}
$$

sharing with the other vertices the LCA and LCH.

In Figure 4, depending on whether just (inner blue line) or Pythagorean (outer red line) intonation is used, the harmonic distance between the fundamental tone $\mathrm{C}$ and the other notes of a 12-tone scale is displayed. The tones compared are those of Table 2, depending on the tuning system. In the next section we discuss the approximation for tones of equal temperament scales.

\footnotetext{
${ }^{6}$ The least squares estimation of $y=k x$ for couples of values $\left\{\left(p_{i}, \alpha p_{i}-\varepsilon_{i}\right)\right\}_{i=1, \ldots, n}, \alpha \in \mathbb{R}, p_{i}=i-1$, for $n>1$ and by assuming $p_{i}$ and $\varepsilon_{i}$ are uncorrelated variables, yields a value

$$
k=\alpha-\bar{\varepsilon} \frac{\sum_{i=1}^{n} p_{i}}{\sum_{i=1}^{n} p_{i}^{2}}=\alpha-\frac{1}{2} \frac{3}{2 n-1} .
$$
}




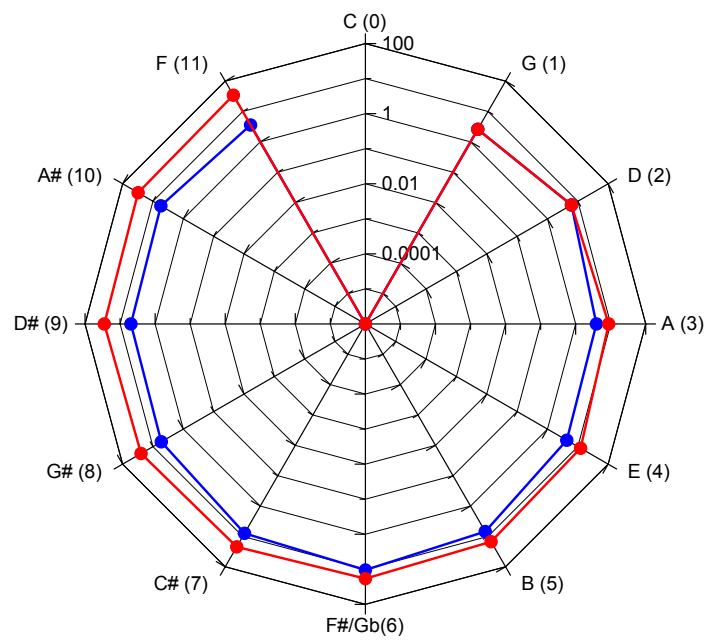

Figure 4. Harmonic distance, in logarithmic scale, from the fundamental tone $\mathrm{C}$ to the other notes of a 12-tone scale: just intonation (inner blue line) and Pythagorean (outer red line). Between brackets, the value of $p$ (index of fifth) for the Pythagorean scale.

(a)

\begin{tabular}{|cccc|}
\hline $\mathrm{A}$ & $\mathrm{E}$ & $\mathrm{B}$ & $\mathrm{TT}$ \\
$2^{\frac{9}{12}}$ & $2^{\frac{4}{12}}$ & $2^{\frac{11}{12}}$ & $2^{\frac{6}{12}}$ \\
\hline $\mathrm{F}$ & $\mathrm{C}$ & $\mathrm{G}$ & $\mathrm{D}$ \\
$2^{\frac{5}{12}}$ & 1 & $2^{\frac{7}{12}}$ & $2^{\frac{2}{12}}$ \\
\hline $\mathrm{C}^{\sharp}$ & $\mathrm{G}^{\sharp}$ & $\mathrm{D}^{\sharp}$ & $\mathrm{A}^{\sharp}$ \\
$2^{\frac{1}{12}}$ & $2^{\frac{8}{12}}$ & $2^{\frac{3}{12}}$ & $2^{\frac{10}{12}}$ \\
\hline
\end{tabular}

(b)

\begin{tabular}{|cccc|}
\hline $\mathrm{A}$ & $\mathrm{E}$ & $\mathrm{B}$ & $\mathrm{F}^{\sharp}$ \\
$\frac{3^{3}}{2^{4}}$ & $\frac{3^{4}}{2^{6}}$ & $\frac{3^{5}}{2^{7}}$ & $\frac{3^{6}}{2^{9}}$ \\
\hline $\mathrm{F}$ & $\mathrm{C}$ & $\mathrm{G}$ & $\mathrm{D}$ \\
$\frac{3^{11}}{2^{17}}$ & 1 & $\frac{3}{2}$ & $\frac{3^{2}}{2^{3}}$ \\
\hline $\mathrm{C}^{\sharp}$ & $\mathrm{G}^{\sharp}$ & $\mathrm{D}^{\sharp}$ & $\mathrm{A}^{\sharp}$ \\
$\frac{3^{7}}{2^{11}}$ & $\frac{3^{8}}{2^{12}}$ & $\frac{3^{9}}{2^{14}}$ & $\frac{3^{10}}{2^{15}}$ \\
\hline
\end{tabular}

(c)

\begin{tabular}{|cccc|}
\hline $\mathrm{A}$ & $\mathrm{E}$ & $\mathrm{B}$ & $\mathrm{G}^{b}$ \\
$\frac{5}{3}$ & $\frac{5}{4}$ & $\frac{15}{8}$ & $\frac{45}{32}$ \\
\hline $\mathrm{F}$ & $\mathrm{C}$ & $\mathrm{G}$ & $\mathrm{D}$ \\
$\frac{4}{3}$ & 1 & $\frac{3}{2}$ & $\frac{9}{8}$ \\
\hline $\mathrm{C}^{\sharp}$ & $\mathrm{G}^{\sharp}$ & $\mathrm{D}^{\sharp}$ & $\mathrm{A}^{\sharp}$ \\
$\frac{16}{15}$ & $\frac{8}{5}$ & $\frac{6}{5}$ & $\frac{9}{5}$ \\
\hline
\end{tabular}

Table 2. Notes of 12-tone scales depending on the tuning system used to compare harmonic distances in chords: (a) 12-TET, (b) Pythagorean tuning, and (c) just intonation.

\section{Equal temperament}

In a $n$-TET scale it is not appropriate to speak of harmonic distance, since the tones are irrational numbers in the form $2^{\frac{i}{n}}, 0 \leq i<n$, such as their ratios. If the elemental interval is approximated by a rational number, i.e., $2^{\frac{1}{n}} \approx \frac{p}{q}$, then the harmonic distance between two tones would be

$$
\Delta_{H}\left(2^{\frac{i}{n}}, 2^{\frac{j}{n}}\right) \approx \Delta_{H}\left(\frac{p^{i}}{q^{i}}, \frac{p^{j}}{q^{j}}\right)=|i-j| \log _{2}(p q),
$$

which is a melodic one dimensional distance.

However, according to Tenney (2015, 280-304), in his 1983 John Cage and the Theory of Harmony, for a scale close to just intonation such as an equally tempered scale, ear tends to resolve towards the closest just intonation harmonic distance. This can be justified on the basis of the categorical perception phenomenon, when stimuli on a physical continuum (in the current case, musical intervals) are placed into a few distinctive categories (Siegel and Siegel 1977; Burns and Ward 1978); although recognition of natural categories for music intervals is exclusive of musically trained subjects. In such a case, in an equal temperament system, when trying to estimate the harmonic dissonance of a set of tones whose interval ratios are slightly different from those of a just intonation system, it seems plausible to take the melodic distance between their LCA and LCH in the just intonation system as an approximation for that of the tempered system. We shall see that, if the respective frequencies are close enough, then it is naturally so, admitting at the outset that such a substitution depends on the listener's cultural background and on the sound sample as well. According to Moore, Peters, and Glasberg (1985), the low 
harmonics $(n<5)$ can be mistuned by about $1-3 \%$ relative to their nominal value and still be perceived as belonging to the same harmonic series. However, the threshold diminishes for higher harmonics and depends also on conditions such as the intensity of the partials and on whether the duration of the sound allows the beatings to be noticed. For instance, in Figure 7 we see that the curve of sensory dissonance for a tetrachord in a 12-TET scale (dashed green curve) is very close to that of just intonation (upper red curve), with relative minima around the notes of the tetrachord and of the respective ratios. The same applies to Pythagorean tuning (not shown).

Let us assume that in a $n$-TET scale two coprime harmonics $p$ and $q$ are approximated by $2^{\frac{P}{n}}$ and $2^{\frac{Q}{n}}$. Thereafter the approach will also depend on the good qualities of the tempered scale. The values $P$ and $Q$ can be estimated from the function nearest integer (NINT) as ${ }^{7}$

$$
P=\operatorname{nint}\left(n \log _{2} p\right) ; \quad Q=\operatorname{nint}\left(n \log _{2} q\right) .
$$

Form the tonal graph of edges $p, q$, starting in the fundamental tone (assumed as 1) with nodes $p^{i} q^{j} ; i, j \in \mathbb{Z}$. Similarly, for the tempered system draw the tonal network (with notes possibly belonging to several octaves) with nodes $i P+j Q$, obtained by taking for each frequency $\nu$ the value $\operatorname{nint}\left(n \log _{2} \nu\right)$. Both graphs, depicted in Figure 5, are isomorphic and, if the estimations provided by equation (52) are good enough, the properties of the graph on the left-hand side are also valid for the other one. The graph on the right-hand side is the Tonnetz, although with regard to harmonic distance the vertices should be divided by $n$.
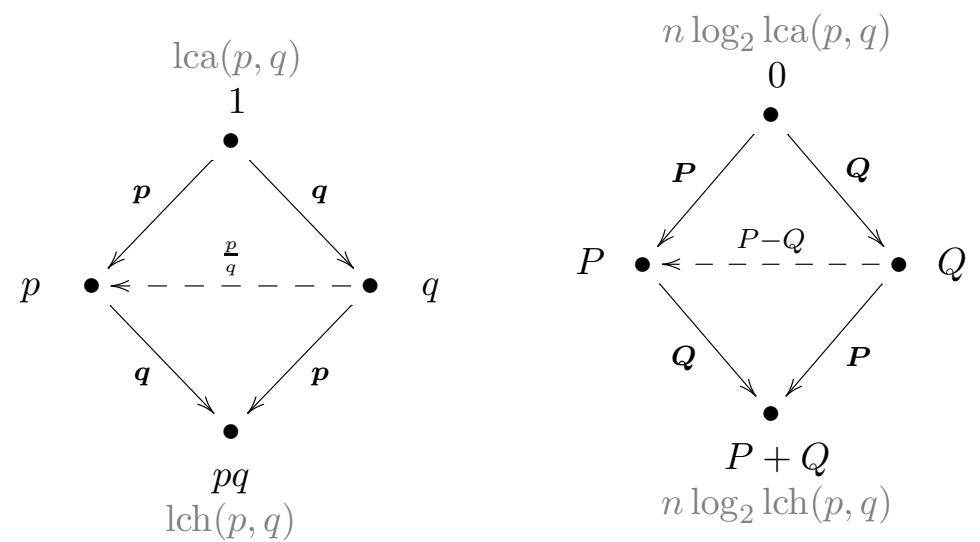

Figure 5. Two isomorphic tonal graphs: on the left, generated by the harmonics $p, q$ and, on the right, generated by the integer intervals $P, Q$ of a $n$-TET system, corresponding to the above harmonics.

Let us assume that the harmonic $p$ is approximated by one note of the $n$-TET scale. Then, the approximation given by equation (52) has an error

$$
\epsilon=2^{\frac{P}{n}}-p
$$

In the graph on left-hand side, the edges will meet in the node $(p+\epsilon) q$ instead of converging to the lowest common harmonic $p q$. In the graph on the right, this tone corresponds to the node $\log _{2}(p+\epsilon)+\log _{2} q$ (regardless of the common factor $n$ shared by all the vertices). Therefore, in the graph on the right, the relative error, referred to just intonation, is

$$
e=\log _{2}\left(1+\frac{\epsilon}{p}\right)
$$

\footnotetext{
7 If $\log _{2} p \approx \frac{a}{b}$ and $\log _{2} q \approx \frac{a^{\prime}}{b^{\prime}}$ are some good rational, irreducible estimates of the respective logarithms, then the minimum number of notes of an equal temperament scale that approximates the previous harmonics is determined by $n=\operatorname{lcm}\left(b, b^{\prime}\right)$. Thus, if the positive integers $\beta, \beta^{\prime}$ are such that $\beta b=\beta^{\prime} b^{\prime}=n$, then the values $P$ and $Q$ are obtained as $P=\operatorname{nint}\left(n \log _{2} p\right)=n \frac{a}{b}=\beta a$ and $Q=\operatorname{nint}\left(n \log _{2} q\right)=n \frac{a^{\prime}}{b^{\prime}}=\beta^{\prime} a^{\prime}$.
} 
If this amount is perceptible by the ear, then we cannot apply the criteria of harmonic dissonance, but if not, the hearing will be guided by the graph on the left. By multiplying $e$ by 1200 we get the error expressed in cents.

For example, if the harmonic $p=3$ is approximated by $2^{\frac{19}{12}}=2.997$ in a 12 -TET scale, the error in cents is $1200 e=-1.9$, i.e., a totally imperceptible amount. By approximating $p=5$ by $2^{\frac{28}{12}}=5.040$, also in a 12 -TET scale, the error is $1200 e=+13.7$ cents, a value about $1 \%$ of the nominal pitch, which, according to Moore, Peters, and Glasberg (1985), depending on its relative intensity can still be perceived as the fifth harmonic. In such a case, the harmonic distance $\Delta_{H}(p, 1)=\log _{2} p$, measured in the graph on the left of Figure 5 , and the value $\frac{P}{n}$, measured in the graph on the right, are very close.

However, for an arbitrary tone of the $n$-TET system, several approximations from a ratio of two coprime harmonics are possible. For example, the frequency $2^{\frac{10}{12}}=1.782$ can be approximated in several ways. A musician trained in an oriental musical tradition could correct it to $\frac{7}{4}=1.750$; a violin player, more used to Pythagorean tuning, may have heard $\frac{16}{9}=1.778$; and someone trained in ancient music could adapt it to $\frac{9}{5}=1.800$. For the harmonics appearing in these ratios, the corresponding approximations in the 12-TET system are

$7 \approx 2^{\frac{1}{12} \operatorname{nint}\left(12 \log _{2} 7\right)}=2^{\frac{34}{12}}, 4=2^{\frac{24}{12}}, 16=2^{\frac{48}{12}}, 9 \approx 2^{\frac{1}{12} \operatorname{nint}\left(12 \log _{2} 9\right)}=2^{\frac{38}{12}}, 5 \approx 2^{\frac{1}{12} \operatorname{nint}\left(12 \log _{2} 5\right)}=2^{\frac{28}{12}}$.

They provide good approximations of the harmonic distances of the respective ratios,

$$
\begin{aligned}
& \Delta_{H}\left(\frac{7}{4}, 1\right)=\Delta_{H}\left(7,2^{2}\right)=\log _{2} 7+2 \log _{2} 2=4.81 \approx \frac{1}{12}(34+24)=4.83 ; \\
& \Delta_{H}\left(\frac{16}{9}, 1\right)=\Delta_{H}\left(2^{4}, 3^{2}\right)=4 \log _{2} 2+2 \log _{2} 3=7.20 \approx \frac{1}{12}(48+38)=7.17 ; \\
& \Delta_{H}\left(\frac{9}{5}, 1\right)=\Delta_{H}\left(3^{2}, 5\right)=2 \log _{2} 3+\log _{2} 5=5.49 \approx \frac{1}{12}(38+28)=5.50 .
\end{aligned}
$$

No mathematical model can predict which pitch correction, if any, will be done. However, once the substitution has been made, we want to determine the conditions making the harmonic distances in both graphs similar.

Let $\nu$ be a FC of the $n$-TET system that is heard as the ratio $\frac{p^{i}}{q^{j}}$, with $p, q$ coprime. Let us assume that, in the $n$-TET system, these values are approximated as $p \approx 2^{\frac{P}{n}}, q \approx 2^{\frac{Q}{n}}$ with $P=\frac{1}{n} \operatorname{nint}\left(n \log _{2} p\right), Q=\frac{1}{n} \operatorname{nint}\left(n \log _{2} q\right)$. We write

$$
\nu=2^{\frac{1}{n}(A-B)} \approx \frac{p^{i}}{q^{j}} ; \quad A=\operatorname{nint}\left(n \log _{2} p^{i}\right), B=\operatorname{nint}\left(n \log _{2} q^{j}\right) .
$$

In order to maintain the similarity of both graphs along $i$ nodes in the $p$-direction and $j$ nodes in the $q$-direction, we must ask $A$ and $B$ to be commensurable with $P$ and $Q$, respectively, i.e., $A=i P$ and $B=j Q$. Therefore, for any harmonic $p$ (and similarly for $q$ ), it must be satisfied

$$
\operatorname{nint}\left(n \log _{2} p^{i}\right)=i \operatorname{nint}\left(n \log _{2} p\right)
$$

(for $i=1$ it is always fulfilled). Then, the harmonic distance will be estimated as

$$
\Delta_{H}\left(\frac{p^{i}}{q^{j}}, 1\right)=\Delta_{H}\left(p^{i}, q^{j}\right)=i \log _{2} p+j \log _{2} q \approx \frac{1}{n}(i P+j Q)=\frac{1}{n}(A+B) .
$$

To determine the values $i$ for which equation (56) is fulfilled, we define the reminder

$$
r(p)=n \log _{2} p-\operatorname{nint}\left(n \log _{2} p\right), \quad|r(p)|<\frac{1}{2} .
$$


Then, $\operatorname{nint}\left(n \log _{2} p^{i}\right)=\operatorname{nint}\left[i \operatorname{nint}\left(n \log _{2} p\right)+i r(p)\right]$, so that ${ }^{8}$

$$
\operatorname{nint}\left(n \log _{2} p^{i}\right)=i \operatorname{nint}\left(n \log _{2} p\right) \Longleftrightarrow|i r(p)|<\frac{1}{2} .
$$

The condition $|i r(p)|<\frac{1}{2}$ provides a maximum value for $i$, and determines the relative error of equation (54) for a pitch approximated by the harmonic $p^{i}$, as

$$
\left|e_{p}\right|=\left|\log _{2} \frac{2^{\frac{i P}{n}}}{2^{\frac{1}{n}(i P+i r(p))}}\right|=\frac{1}{n}|i r(p)|<\frac{1}{2 n} .
$$

Then, for a 12-TET system, the maximum relative error allowed is $2.9 \%$ of the nominal pitch, similar to the maximum perceptive relative errors given by Moore, Peters, and Glasberg (1985). In the end, for the approximation $\nu \approx \frac{p^{i}}{q^{j}}$, the relative error $e_{\nu}$ satisfies

$$
\left|e_{\nu}\right|=\frac{1}{n}|\operatorname{ir}(p)+j r(q)|<\frac{1}{n} .
$$

the same as the error in estimating equation (57). The approximation will be valid as long as the perceptive relative errors are lower than the mathematical relative errors.

It is also possible to give a bound for the difference of the harmonic distances $\Delta_{H}$ and $\Delta_{H}^{\prime}$ from two different estimations of the value $2^{\frac{x}{n}}$, with $x=A-B=A^{\prime}-B^{\prime}$. Assume the pitch is approximated by the irreducible fractions $\frac{a}{b}$ and $\frac{a^{\prime}}{b^{\prime}}$. According to equation (57), the harmonic dissonances are estimated as $\Delta_{H}=\frac{1}{n}(A+B)=\frac{1}{n}(x+2 B)$ and $\Delta_{H}^{\prime}=\frac{1}{n}\left(A^{\prime}+B^{\prime}\right)=\frac{1}{n}\left(x+2 B^{\prime}\right)$. Hence, $\Delta_{H}-\Delta_{H}^{\prime}=\frac{2}{n}\left(B-B^{\prime}\right)$. Owing to the equations (55) and (58), we may write $B=n \log _{2} b-\rho$ and $B^{\prime}=n \log _{2} b^{\prime}-\rho^{\prime}$, with $|\rho|,\left|\rho^{\prime}\right|<\frac{1}{2}$. Then,

$$
\left|\Delta_{H}-\Delta_{H}^{\prime}\right| \leq 2\left(\left|\log _{2} \frac{b}{b^{\prime}}\right|+\frac{|\rho|+\left|\rho^{\prime}\right|}{n}\right)<2\left|\log _{2} \frac{b}{b^{\prime}}\right|+\frac{2}{n},
$$

which is a value depending on the denominators of the ratios: the closer they are, the lower the difference. For instance, the ratios $\frac{16}{9}, \frac{7}{4}$ of the previous examples give a maximum difference of the dissonances of $2\left|\log _{2} \frac{9}{4}\right|+\frac{2}{12}=2.51$, which provides a good limit for the actual difference, which is 2.39 .

The procedure is straightforward generalised to more than two tones. As an example, we consider the set of FCs $\left\{\nu_{i}\right\}_{i \in I}$ of a $n$-TET system and define the sets and values ${ }^{9}$

$$
\Lambda=\left\{\lambda_{i}=\frac{p_{i}^{\alpha_{i}}}{q_{i}^{\beta_{i}}} \approx \nu_{i}\right\}, \Pi=\left\{p_{i}^{\alpha_{i}}\right\}, \Theta=\left\{q_{i}^{\beta_{i}}\right\} ; \quad P_{i}=\operatorname{nint}\left(n \log _{2} p_{i}\right), Q_{i}=\operatorname{nint}\left(n \log _{2} q_{i}\right)
$$

satisfying $\nu_{i}=2^{\frac{1}{n}\left(\alpha_{i} P_{i}-\beta_{i} Q_{i}\right)} ;$ under the condition of indiscernible relative errors $\left|\alpha_{i} r\left(p_{i}\right)\right|,\left|\beta_{i} r\left(q_{i}\right)\right|$. Then, according to Section 6, the harmonic dissonance of the FCs $\nu_{i}$ can be approximated from that of $\Lambda$ as

$$
\Delta_{H}(\Lambda)=\log _{2} \frac{\operatorname{lcm}(\Pi)}{\operatorname{gcd}(\Pi)}+\log _{2} \frac{\operatorname{lcm}(\Theta)}{\operatorname{gcd}(\Theta)}=\sum_{i \in I} \log _{2} p_{i}^{x_{i}}+\log _{2} q_{i}^{y_{i}} \approx \frac{1}{n} \sum_{i \in I} x_{i} P_{i}+y_{i} Q_{i}
$$

for certain non-negative integers $x_{i} \leq \alpha_{i}$ and $y_{i} \leq \beta_{i}$.

\footnotetext{
${ }^{8}$ Notice that $i \operatorname{nint}\left(n \log _{2} p\right)=\operatorname{nint}\left(i \operatorname{nint}\left(n \log _{2} p\right)\right)$.

${ }^{9}$ The case where each pitch $\nu$ is approximated by a ratio $\lambda=\frac{p_{1}^{\alpha_{1}} \ldots p_{k}^{\alpha_{k}}}{q_{1}^{\beta_{1}} \ldots q_{l}^{\beta_{l}}}$, by taking into account equations (39) and (41) has a similar although cumbersome treatment.
} 


\section{Trichords}

In the last Section we shall compare different concepts of dissonance and, in particular, we shall evaluate the harmonic dissonance of the neighbouring chords on the Tonnetz in order to see if there is some correlation with their the geometrical distribution and with the most efficient voice-leading paths. For this reason, some basic facts about such a topography of diatonic triads are described.

Notice that each triangle of Figure 5 form a trichord and that the harmonic dissonance of the triplets formed by the vertices $\{1, p, q\}$ and $\{p, q, p q\}$ is the same. However, the chords formed by their FCs have, in general, different harmonic dissonance. In the next section we shall calculate harmonic dissonances of chords composed of pure tones and of FCs as well. For example, the $\mathrm{C}$ major chord composed of FCs has harmonic dissonance $\Delta_{H}\left(1, \frac{3}{2}, \frac{5}{4}\right)=5.91$, while when it is composed of pure harmonics has the lowest value $\Delta_{H}(1,3,5)=3.91$.

If we deal with pitch classes, on the right-hand side graph, the values $P, Q$ and their combinations take values in $\mathbb{Z}_{n}$. The ratio between the intermediate nodes $p$ and $q$ on the left-hand side graph is $p / q$, while the interval difference between the respective nodes on the right-hand side graph is $(P-Q) \bmod n$. We shall take clockwise direction along edges of the triangles as positive direction of the arrows. Then, the nodes $\{0, P, Q\}$ and $\{P, Q,(P+Q) \bmod n\}$ of adjacent triangles, and their counterparts of Figure 5, form two trichords that, at least share two tones. In such a case, the bidimensional arrangement of the trichords is a criterion typically used to define a topography of chords. This is the point of view adopted in neo-Riemannian music theory (e.g., Lewin 1987; Cohn 1998; Tymoczko 2006).

Let us consider, for instance, the major triads composed of pitch classes of the 12-TET scale. We shall refer to the tonal graph of Figure 6 around the $\mathrm{C}$ major chord $M_{1}=(\mathrm{C}, \mathrm{E}, \mathrm{G})$ generated by the two lowest prime harmonics other than the fundamental. The pitch class of the third harmonic -the fifth- corresponds to a note 7 semitones away from the fundamental, and the class of the fifth harmonic - the major third- is 4 semitones far away from the fundamental. The ratio $\frac{6}{5}$ between these tones in the interval $[1,2)$ provides the minor third, at 3 semitones from the fundamental. Thus, the multiplicative factors between FCs are associated with translations in the logarithmic equal temperament scale.

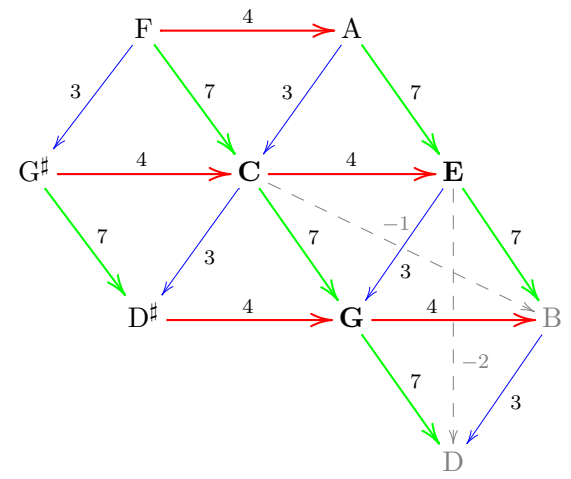

Figure 6. Tonal graph centred in the note $\mathrm{C}$, generated by the tonal functions of the third and fifth harmonics. The black notes belong to major and minor triads that contain the note C. Arrows indicate translations in number of semitones of the 12-TET scale.

In this figure, the triangles shaped as $\nabla$ are major chords and those shaped as $\Delta$ are minor chords. If we focus in the chords containing the note $\mathrm{C}$, we see that adjacent chords share two notes, one of them C. This is the domain or chord cell of the note C, which contains, in regard with the composition of their intervals, both modes of chords, three major chords with intervals $[4,3,5]=[4,3,-7]$ (measured in $\mathbb{Z}_{12}$ ) according to a clockwise-oriented cyclic path, and three 
minor chords with intervals $[3,4,5]=[3,4,-7]$ according to a counterclockwise-oriented cyclic path. The major chords are related by a simultaneous translation of their notes from another major chord of 7, 4 and 3 semitones along the respective arrows, or of their opposite intervals 5 , 8 and 9 in the opposite sense. The same applies to minor chords. By translations, both modes cannot be mixed. If the $\mathrm{F}$ major chord $M_{\frac{4}{3}}=(\mathrm{F}, \mathrm{A}, \mathrm{C})$ is translated one fifth $(7$ semitones along the green arrow) we reach the $\mathrm{C}$ major chord $M_{1}=(\mathrm{C}, \mathrm{E}, \mathrm{G})$. This is the direction and sense of the harmonically closest major chord, as will be quantified in the following section. The reverse translation, i.e., a fourth from $\mathrm{C}$ major to $\mathrm{F}$ major, leads to the second harmonically closest major chord. If we move a major third (4 semitones along the red arrow), we reach the third harmonically closest major chord.

The only chord sharing two notes with the $\mathrm{C}$ major chord (in boldface) that does not belong to the chord cell of $\mathrm{C}$ is the $\mathrm{E}$ minor chord $m_{\frac{5}{4}}=(\mathrm{E}, \mathrm{G}, \mathrm{B})$. In the next section we shall see that this is the chord of the tonal graph that is at minimum harmonic distance from the $\mathrm{C}$ major chord. This minor chord introduces the new note B, the seventh degree of the diatonic major scale, i.e., the leading tone. To connect the cell $\mathrm{C}$ with a cell of a note not contained in the former through the $\mathrm{C}$ major chord (with a chord that at least shares one note), the less harmonically dissonant way do it is through the E minor chord, which belongs to the cell of the leading tone $\mathrm{B}$. If it is limited to the use of major chords, then the chord harmonically closer to $\mathrm{C}$ is the $\mathrm{G}$ major chord $M_{\frac{3}{2}}=(\mathrm{G}, \mathrm{B}, \mathrm{D})$, which also contains the leading tone and belongs to the cell D. The subject of voice leading and chord geometry can be deepened from comprehensive studies such as Douthett and Steinbach (1998); Tymoczko (2006); Callender, Quinn, and Tymoczko (2008).

\section{Discussion}

Several examples will illustrate different concepts of dissonance. For chords, we compare the harmonic dissonance $\Delta_{H}$ of equation (41) to the sensory dissonance ${ }^{10} \Delta_{S}$. For instance, for the major tetrachord $\left\{1, \frac{5}{4}, \frac{3}{2}, 2\right\}$, referred to a fundamental frequency of $500 \mathrm{~Hz}$ and with notes of the same intensity, in Figure 7 (upper red curve) we determine the translated tetrachords (maintaining the same interval structure) more consonant with the above ones. The factors $r$ corresponding to relative minima of dissonance match certain fractions of the fundamental frequency, that vary depending on the spectrum and on the base frequency. It is also shown the nearby curve for the chord in the 12-TET tuning system (dashed green line). For a triad $\left\{1, \frac{5}{4}, \frac{3}{2}\right\}$, without doubling the fundamental frequency in the upper octave, we get the lower curve in blue, where three relative minima of dissonance have been lost. Therefore, the sensory dissonance of a chord measures the roughness sensation of any set of real frequencies, not necessarily in rational proportions. When the spectrum consists of commensurable tones, the minima of sensory dissonance take place on rational frequencies, although, depending on the spectrum, the hierarchy of dissonances may vary with regard to Table 1 . Hence, this model is useful when the timbre of an instrument is known, i.e., if the vibration modes sounding when a note is played are known as well as their hierarchy. Curves of sensory dissonance show few relative minima when they are computed within a small interval of frequencies, however are very useful when spectrum is

${ }^{10}$ According to Sethares $(1998,345)$ it is computed as

$$
\Delta_{S}(F)=\frac{1}{2} \sum_{i \neq j} \min \left(a_{i}, a_{j}\right)\left(e^{-b_{1} s_{i j}\left|\nu_{i}-\nu_{j}\right|}-e^{-b_{2} s_{i j}\left|\nu_{i}-\nu_{j}\right|}\right)
$$

with $s_{i j}=\frac{0.24}{0.0207 \min \left(\nu_{i}, \nu_{j}\right)+18.96}$ and the constants $b_{1}=3.51, b_{2}=-5.75$. The above formula is useful to measure the sensation of dissonance produced by the union $F \cup r F$ of two spectra, composed of tones that maintain mutually a frequency ratio $r$, since it allows to detect which are the ratios producing a minimum of sensory dissonance. Generally, since the scale of dissonance is arbitrary, it will be appropriate to compare relative dissonances and, in particular, more than the value itself, it is important to know where the relative minima are placed. The curve of equation (65), for a set $F$ of $n$ tones, has up to $2 n(n-1)$ relative minima, corresponding to ratios that provide a greater sensation of consonance. 


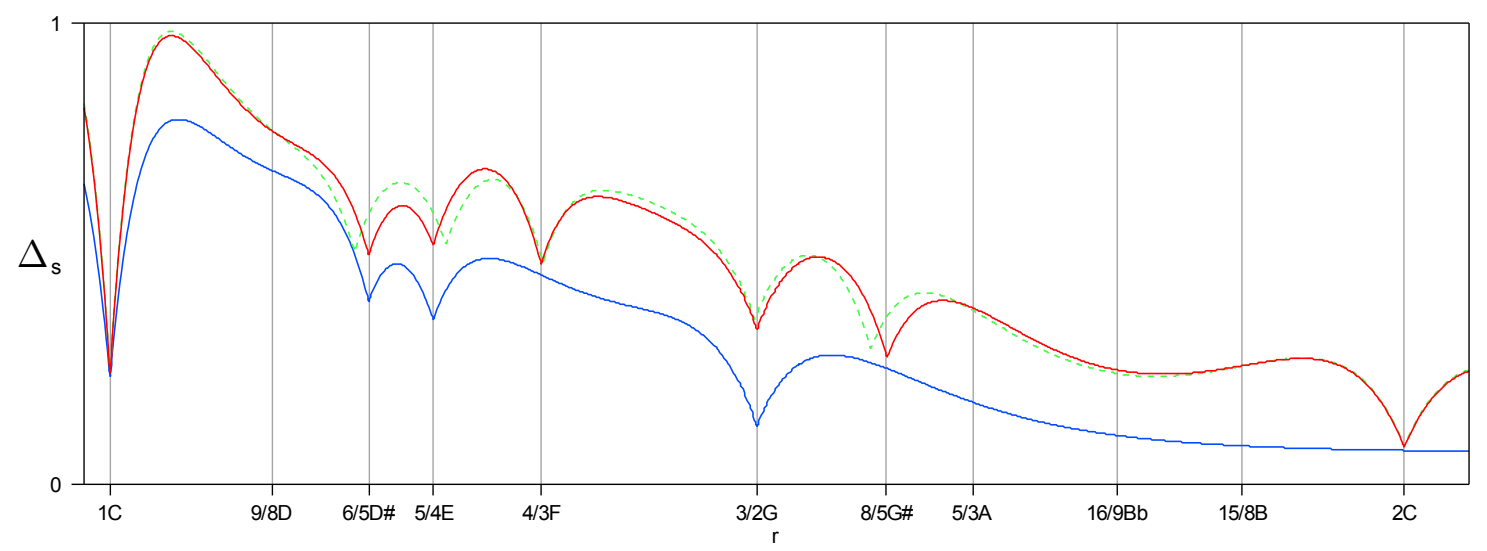

Figure 7. Sensory dissonance in terms of the factor $r$ for chords $F$ and $r F$, referred to a frequency of $500 \mathrm{~Hz}$, with $F=\left\{1, \frac{5}{4}, \frac{3}{2}, 2\right\}$ (upper red curve); $F=\left\{1,2^{\frac{1}{3}}, 2^{\frac{7}{12}}, 2\right\}$ (dashed green curve); and $F=\left\{1, \frac{5}{4}, \frac{3}{2}\right\}$ (lower blue curve).

sufficiently dispersed. Therefore, it is not appropriate to characterise chords consisting of FCs.

In Figure 8 we show some examples for several chords in relation to the $\mathrm{C}$ major chord of tonic. First, we compare the harmonic dissonance to the above sensory dissonance (scaled and with fundamental at $500 \mathrm{~Hz}$ ) for chords consisting of 3 and 4 tones in root position. The spectrum for sensory dissonance is taken as the union of the tones of the chord pair with the same amplitudes. The graph is ordered according to trichords (and tetrachords) with increasing $\Delta_{H}$.

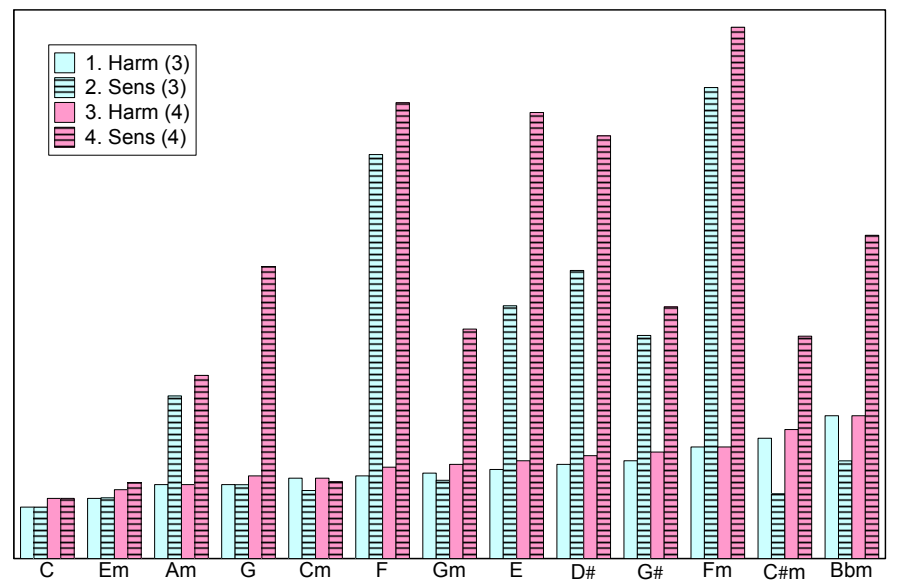

Figure 8. Harmonic dissonance (Harm.) and sensory dissonance (Sens.) for chords in root position composed of 3 and 4 tones.

It is worth noticing that, when the fundamental is doubled, a perceptible change of sensory dissonance takes place, especially for the chords $C^{\sharp} \mathrm{m}, \mathrm{G}, \mathrm{Gm}$ and $\mathrm{B}^{b} \mathrm{~m}$.

Therefore, sensory and harmonic dissonances do not evaluate the same aspect of sound, although, depending on the spectrum we may find certain degrees of correlation. While harmonic dissonance basically depends on the lowest harmonics, sensory dissonance also evaluates the beatings produced by the whole set of harmonics (those involved in the spectrum), so that if they are weighted with amplitudes similar to their fundamental, their interferences will produce a great amount of beatings.

Now we compare the harmonic dissonance between tones in terms of the tuning system. Figure 9 shows harmonic dissonances between the $\mathrm{C}$ major chord and the chords of the previous chart. Blue bars (each third bar in the chart) are for just intonation chords composed of pitch classes. This has been the criterion for sorting the chords. If these tones are interpreted as pitches, then the chord corresponds to the inversion indicated within brackets. If this is calculated for 


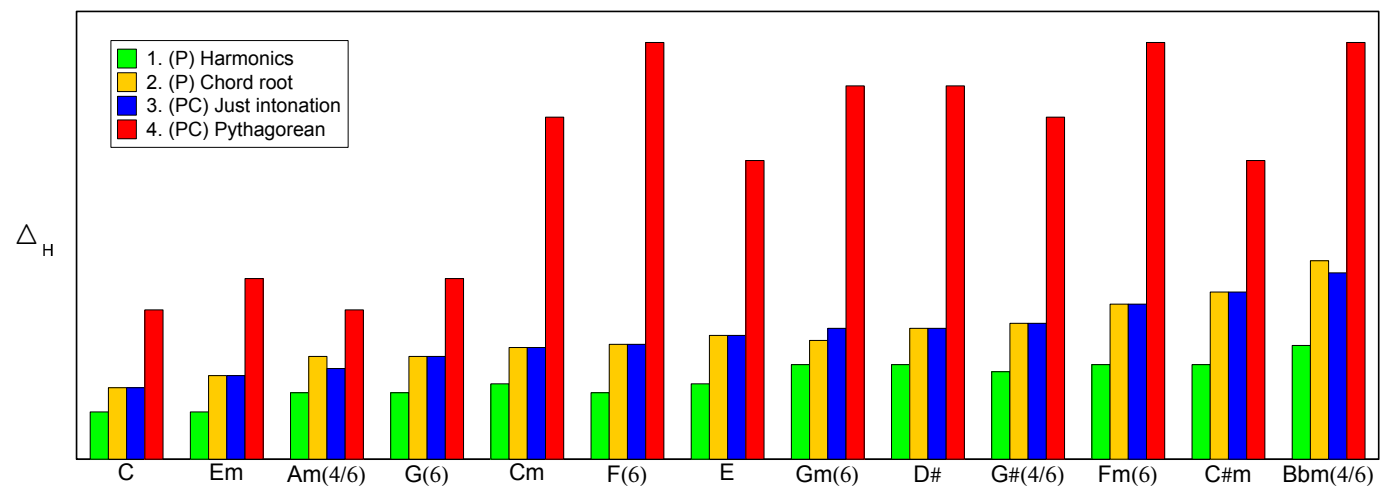

Figure 9. Harmonic dissonance between the major chord of tonic and other chords. For pitch classes (PC): just intonation (blue) and Pythagorean tuning (red). For pitches (P): chords consisting of pure harmonics (green) and chords in root position (orange). Brackets indicate the chord inversion when pitches are considered instead of pitch classes.

chords in root position, the harmonic dissonance slightly changes, as shown by the orange bars. If the chords consist of pure harmonics, i.e., expanding to more than one octave, the harmonic dissonance drops off, as shown by the green bars. For Pythagorean tuning with pitch classes, the harmonic dissonance is depicted by red bars and increases dramatically.

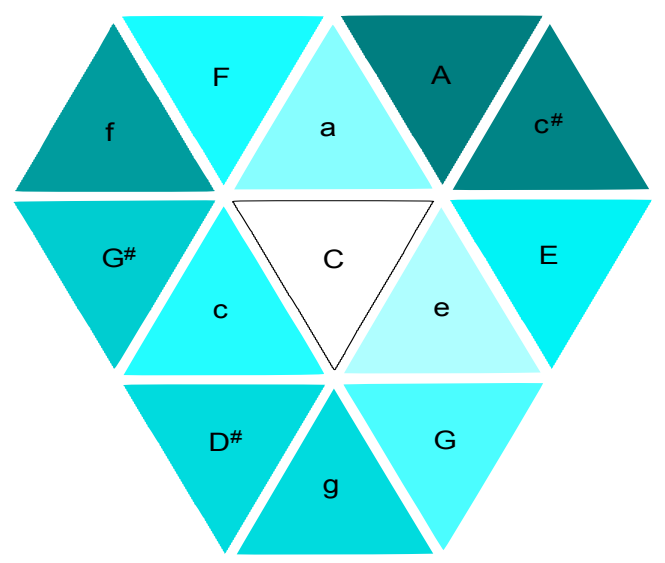

Figure 10. Harmonic dissonance for chords composed of pitch classes from the tonal graph Figure 6 in relation to the $\mathrm{C}$ major chord. The darker, the more dissonant. In lowercase for minor chords.

Finally, the harmonic dissonance of the chords in the tonal graph of Figure 6 around the C major chord is represented in Figure 10, by painting darker the chords more harmonically dissonant with the central chord. The calculation is for pitch classes. The major chords harmonically closest to $\mathrm{C}$ major are the major chords $\mathrm{G}$ and $\mathrm{F}$, although the minor chords Em and Am are even closer. The chord with the lowest harmonic dissonance with $\mathrm{C}$ is Em. This chord contains the leading tone, which does not belong to any chord containing the note $\mathrm{C}$, also shared by the $\mathrm{G}$ major chord. Starting from the $\mathrm{C}$ major chord, the most harmonically stable modulations are towards the minor chords Em and Am, and, in second place, towards the major chords $\mathrm{G}$ and $\mathrm{F}$. As Am and $\mathrm{F}$ also contain the note $\mathrm{C}$, the less dissonant modulation seeking to change the fundamental must be done towards $\mathrm{Em}$ or $\mathrm{G}$. The $\mathrm{Cm}$ chord is adjacent to the $\mathrm{C}$ major chord, although is harmonically more dissonant than the $\mathrm{G}$ major chord (and than $\mathrm{F}$ if pure harmonics are considered). Therefore, the proximity of chords in the chord network does not match the criterion of harmonic distance, although harmonic consonance is correlated with most efficient voice-leading chains between diatonic triads (Tymoczko 2006; Callender, Quinn, and Tymoczko 2008) obtained from the neo-Riemannian transformations L (in this example, C major to Am) and $\mathrm{R}$ ( $\mathrm{C}$ major to $\mathrm{Em}$ ), while the $\mathrm{P}$ transformation (C major to $\mathrm{Cm}$ ) is less efficient and less consonant. 
Harmonic dissonance depends on frequency distances. Proximity of chords is based on their distribution on the Tonnetz according to an equal temperament system, where pitch classes are considered instead of pitches. Pitch classes depend on the key dictated by the fundamental. Chord inversions have different harmonic dissonances. Therefore, in Figure 10 if one changes the fundamental, the relative contrast between chords will vary but not their relative position.

In summary, the harmonic dissonance of a set of commensurable pitches measures the melodic distance between their LCA and LCH on a tonal graph, the former, the frequency of the common fundamental, the latter, the frequency that summons all these pitches as subharmonics. Tones associated with a lower harmonic distance in the tonal graph tend to be heard as being in a consonant relation to each other, while tones represented by a greater harmonic distance are heard as mutually dissonant.

\section{Acknowledgments}

I wish to thank two anonymous referees for very useful and constructive comments that improved the manuscript significantly, and Editors Thomas Fiore and Emmanuel Amiot for valuable remarks and comments. I would also like to thank Sara Cubarsi for helpful discussions about Tenney's theory of consonance.

\section{References}

Burns, E.M., and W.D. Ward. 1978. "Categorical Perception - Phenomenon Or Epiphenomenon: Evidence from Experiments in the Perception of Melodic Musical Intervals." The Journal of the Acoustical Society of America 63: 456.

Callender, C., I. Quinn, and D. Tymoczko. 2008. "Generalized Voice-Leading Spaces." Science 320: 346.

Cohen, H.F. 1985. "Music As a Test-case." Stud. Hist. Phil. Sci. 16 (4): 351.

Cohn, R. 1998. "An Introduction to Neo-Riemannian Theory: A Survey and Historical Perspective." Journal of Music Theory 42 (2): 167.

Douthett, J., and P. Steinbach. 1998. "Parsimonious Graphs: A Study in Parsimony, Contextual Transformation, and Modes of Limited Transposition." Journal of Music Theory 42 (2): 241.

Ebeling, M. 2008. "Neuronal periodicity detection as a basis for the perception of consonance: A mathematical model of tonal fusion." The Journal of the Acoustical Society of America 124: 2320.

Fastl, H., and E. Zwicker. 2007. Psychoacoustics: facts and models. 3rd ed. Berlin: Springer.

Helmholtz, H.v. 1863. On the Sensations of Tone as a Physiological Basis for the Theory of Music. London: Longman's, Green, and Company. English translation by Ellis, A.J. 1875, https://archive.org/details/onsensationsofto00helmrich.

Langner, G. 2015. The Neural Code of Pitch and Harmony. Cambridge: Cambridge University Press.

Lewin, D. 1987. Generalized Musical Intervals and Transformations. New Haven, CT: Yale University Press.

Mazzola, G., S. Göller, and S. Müller. 2012. The Topos of Music: Geometric Logic of Concepts, Theory, and Performance. SpringerLink: Bücher. Basel: Birkhäuser.

Mickens, R.E. 1981. An Introduction to Nonlinear Oscillations. Cambridge: Cambridge University Press.

Moore, B.C.J., R.W. Peters, and B.C. Glasberg. 1985. "Thresholds for the Detection of Inharmonicity in Complex Tones." Journal of the Acoustical Society of America 77: 1861.

Plomp, R., and W.J.M. Levelt. 1965. "Tonal Consonance and Critical Bandwidth." Journal of the Acoustical Society of America 38: 548.

Sethares, W.A. 1998. Tuning, Timbre, Spectrum, Scale. London: Springer-Verlag.

Siegel, J.A., and W. Siegel. 1977. "Categorical Perception of Tonal Intervals: Musicians Can't Tell Sharp from Flat." Perception \& Psychophysics 21: 399.

Stolzenburg, F. 2015. "Harmony Perception by Periodicity Detection." Journal of Mathematics and Music 9 (3): 215.

Stumpf, C. 1890. Tonpsychologie. Hilversum: Knuf. Reprinted 1965.

Tenney, J. 1988. A History of Consonance and Dissonance. New York: Excelsior Music Publishing Co.

Tenney, J. 2015. From Scratch. Urbana, Illinois: University of Illinois Press.

Tymoczko, D. 2006. "The Geometry of Musical Chords." Science 313: 72. 